\title{
Use of routinely collected data on psychiatric in-patient care
}

\author{
Gyles Glover
}

Abstract Since the start of the National Health Service, data have been collected on admissions to psychiatric inpatient units, first as the Mental Health Enquiry, then as part of Hospital Episode Statistics. Some details have changed but many have stayed remarkably consistent. Published literature on the wide range of research and policy work undertaken using this data source is reviewed. Early work was central to the government's deinstitutionalisation policy in the early 1960s. Subsequent studies cover a wide range of epidemiological and health services research issues. A new statistical base, the Mental Health Minimum Data Set, covering individuals receiving all types of health care is currently being set up. This will supplement (but not replace) admission statistics.

The Department of Health is introducing a new system for collecting data about National Health Service (NHS) mental health care, the Mental Health Minimum Data Set (Glover, 2000). Individual records will be constructed about each period of care received by a patient in the specialist mental health services. In designing the system, a review was undertaken of how in-patient statistics, the only source currently collected in such detail, have been used. The aim was to identify features necessary to make the new source applicable in a similar manner.

Here I give a brief history of the collection of admission data (outlined in Box 1) and discuss research studies that used this information.

\section{The history of NHS mental hospital admission data}

Statistical gathering of data about care activity in the early NHS was undertaken by the General Register Office. The exercise was considered to have three purposes: understanding the extent and distribution of health care needs in the new NHS; studying variations in effectiveness between hospitals; and mapping out the new challenges for public health (Registrar General, 1953).

Data collection was originally carried out using an entirely manual system. Cards for the Mental

\section{Box 1 Routine in-patient statistics}

Records of all psychiatric admissions have been collated and reported since 1949

Data from 1974 are extant in computerised form

Data were collected by the General Register Office until 1960 and then by the Ministry of Health and its successors

There are two breaks in the sequence, 19601963 and 1987-1990, each reflecting disruption associated with new collection arrangements

Health Enquiry were issued to hospitals for completion about each admission. These were returned to a national coding centre, where they were checked and collated, to allow patient-based analysis. In 1960, the task was transferred to the Ministry of Health, where the exercise was considered to have a more limited, managerial, role (Ministry of Health, 1969). Patient linkage was discontinued and the scope of the data was reduced.

The system was reviewed following the NHS reorganisation of 1974 and again in the 1980s as part of the Korner review of health service information (Korner, 1982). This last review consolidated mental hospital data into the new general format for admission data, called Hospital Episode Statistics. From this point, hospitals were required

Gyles Glover is Professor of Public Mental Health at the University of Durham (Centre for Public Mental Health, Elvet Riverside II, New Elvet, Durham DH1 3JT, UK. E-mail: Gyles.Glover@durham.ac.uk). He has worked extensively at the Department of Health and, at the time that this work was undertaken, he was partly funded by the Department. He has researched routine data sources describing mental health services and their activity. He is responsible for the annual mental health services mapping exercises in England and designed the new Mental Health Minimum Data Set, which came into full operation from April 2003. 
to submit data in an electronic format. This structure is still in use. The changes in 1960 and 1987 were each associated with about 3 years of data loss. Data from 1974 to the present day is extant in computerised form.

\section{Search methods}

I used government publications to identify work undertaken before the start of computerised literature indexing. For studies from 1966, a Medline search was undertaken using all appropriate combinations of the following keys: information science, government, health services need and demand, mental disorders, psychiatric services, psychiatric department - hospital, public health, and statistics. Title and abstract searches were undertaken for the text phrases KORNER, MENTAL HEALTH ENQUIRY, HOSPITAL EPISODE STATISTICS, ROUTINE DATA and ROUTINE STATISTICS. The search was restricted to English language papers and titles and abstracts were screened to identify relevant papers.

\section{Research by the General Register Office}

The principal publications from the General Register Office period comprise six reports, covering the period from 1949 to 1959 in 1-, 2- or 3-year intervals (Registrar General, 1953, 1955, 1958, 1960,1961, 1962). Two supplementary reports were produced and these are described below. These reports contain the most analytical work undertaken in the history of English national mental health statistics. Both technically and conceptually innovative, they were highly influential in shaping the pattern of mental health policy over the subsequent decades. Given that they were produced without the assistance of computerised data handling, they represent a level of government commitment to the development of an evidencebase for mental health policy which, by modern standards, is astonishing.

Most of the publications based on these early data are better considered as routine government statistical publications than research and are thus beyond the scope of this review. However, following the establishment of the patient-based register in 1954, two major studies were undertaken which undoubtedly fall within the research domain.

The first documented the patterns of illness across social classes and occupational groups (Registrar General, 1958). Record linkage enabled the authors to avoid double counting of patients despite the emerging pattern of early discharge and multiple readmission. The broad picture was that admission for most types of mental illness was more common among manual than non-manual social classes. Among non-manual workers, rates of illness were generally higher in class I than in class II, whereas among manual workers, admissions were particularly concentrated in class V. Schizophrenia showed this pattern to a greater extent than did manic-depressive illness. Alcohol-related problems stood out, however, being very much more prominent in older men of working age in social class I.

From the very large tabulations produced by combining data from the 5 years 1949-1953, the authors sought to identify within each social class occupations with consistently high or low first-admission rates for schizophrenia, manicdepressive illness and other diagnoses. They concluded that there were particular risks associated with high standards of education, clerical and domestic work and the catering industry.

The trend tables that were developed in the regular reports of the 1950s demonstrated striking increases in both first-admission and readmission rates, with the latter becoming an increasing proportion of the total. Much of the increase in first admissions arose among elderly people admitted for dementia. At the same time, stay lengths were falling and the overall numbers of occupied beds began to drop from 1954.

The second major study explored these changing patterns of hospitalisation. A cohort of all patients first admitted in 1954 and 1955 was tracked (Brooke, 1963). This showed that, although readmission rates were rising, both recruitment to long-stay status and aggregate bed-use by repeatedly admitted individual patients were declining. In a key paper, published in the Lancet, Tooth \& Brooke (1961) spelt out the policy implication of these findings: the demand for mental hospital care in the foreseeable future was likely to decline to such an extent that the structure of the asylum system would cease to be viable. Describing how the use of hospitalisation was changing, they identified three groups of patients: the 'old long-stay', 'new long-stay' and 'short admission'. They thought that most of the patients in the old long-stay group would probably not have been hospitalised at all or would not have become long-stay patients had their patient careers begun under the treatment practices of the late 1950s. They developed a statistical model of the rate of attrition in the numbers of these patients. Despite contemporary treatment methods, they thought that a small number of new long-stay patients would continue to need long-term hospital care and that the number of individuals requiring short admissions would remain the same over time. They used cohort methods to estimate the numbers in each group. 
This work underpinned the deinstitutionalisation policy set out in Enoch Powell's famous 'water tower' speech and the estimates of psychiatric bed numbers likely to be required in the new District General Hospitals described in the Hospital Plan for England and Wales (Department of Health, 1962).

\section{Reliability}

Cooper (1967) examined the quality of Mental Health Enquiry diagnoses in a careful and detailed study of 293 patients who were indicated on the national admissions register of the mid-1950s to have been admitted at least four times within 2 years. By reference to hospital case notes, he explored whether changes in Mental Health Enquiry diagnoses reflected real clinical changes or erratic coding. Roughly one-third (92) showed a change of diagnosis and in only one-third (32) of these cases did the author consider it justified. His analysis of the case notes suggested that greater standardisation was needed, as much in clinical practice as in the process of recording and coding.

Two more minor studies examined the quality of Mental Health Enquiry data. Gandy (1979), in Leicestershire, undertook a validation study to examine the reason why the picture given of two local mental illness units differed in ways which did not reflect local understanding about their operation. He drew attention to the local data collection arrangements, which resulted in systematic differences in the completeness of some data items and poor accuracy in others. Diagnosis was missing from $19 \%$ of cases and differed from a case note review diagnosis in a further $14 \%$. Forster \& Madhevan (1981) found more satisfactory data in a study in the north-east of England. The referral source showed $20.5 \%$ errors, diagnosis $14.8 \%$ and outcome $10.8 \%$.

\section{Interpretation}

Studies of the epidemiology of major mental illness have used first-admission rates as an approximation of incidence. This makes two assumptions. First, that nearly everyone with a major mental illness will be admitted to hospital at some stage. Second, that the diagnoses at their first admission will include the illness they are eventually recognised to have. Anecdotal experience suggests that the second assumption, at least, is often wrong. Goldacre et al (1994), working at the Oxford Record Linkage study, used their person-based linkage and wider diagnostic data on all patients to explore the validity of both, taking as an example secondary mental health services for schizophrenia. They found that only $70 \%$ were hospitalised at any time in a 12-year period and that only $83.7 \%$ of men and $68.1 \%$ of women with any admission for schizophrenia received this diagnosis in their first admission.

\section{Epidemiological studies}

Epidemiology is the study of disease in populations. Here, Mental Health Enquiry and Hospital Episode Statistics data have much to offer. Data are effectively free. Within their scope, coverage is near to being comprehensive and the number of cases recorded is very large. This allows the study of patterns of service-use at a level of detail not possible in studies dependent on collection of new data.

\section{Marital status and mental illness}

Price et al (1971) reported on the link between marital status and mental hospital admission. In an illfocused paper, they surveyed marital profiles for patients admitted with mental illnesses of all types. They discussed their findings from such diverse perspectives as the implications of low fertility for genetic theories on the propagation of schizophrenia and the fraction of mental illness in the population that could be attributed to widowhood.

\section{The incidence of schizophrenia}

Der et al (1990) explored trends in the first-admission rate for schizophrenia to see if reports of a recent decline in incidence from smaller case-register studies in Scotland, Denmark, Australia and New Zealand were mirrored nationally. Using a combination of published data and special tabulations, they were able to study the period from 1952 to 1986 . First-admission rates seemed roughly stable at about 18 per 100000 population until the mid-1960s and showed a sharp decline from the mid-1970s, levelling in the early 1980s at about 8.5 per 100000 . Problems with data from the late 1960s made it impossible to date the start of the decline accurately. No other diagnostic category showed a reciprocal rise, and this might suggest simple diagnostic shifts.

\section{The outcome of treatment of mania}

Symonds \& Williams (1981) used Mental Health Enquiry data to look at the impact of lithium in the treatment of manic-depressive disorder. Since the introduction of lithium in 1968, there had been a rapid increase in its widespread use and these 
authors looked for a reduction in numbers of hospital admissions for manic-depressive psychosis as evidence of its efficacy. They did not find it. The clear underlying downward trends in all first admissions for both genders was not reflected for this diagnosis and, for women, the number of readmissions was rising both absolutely and as a proportion of all admissions. The authors considered a range of explanations, from changes in the nature of the illness or decreasing societal tolerance to a tendency of psychiatrists to diagnose problems for which they have remedies.

\section{Patterns of mental illness in immigrants}

Small studies in the 1960s and early 1970s had suggested that mental illness was more common in immigrants than in indigenous people in the UK. These, however, had lacked the numbers of cases needed to make adequate allowance for differences in the age structure of the population. Cochrane (1977) in Birmingham used Mental Health Enquiry data for the population census year 1971 to examine this issue. He confirmed the observation that crude all-causes admission rates for almost all groups born outside England and Wales were higher than those for locally born individuals, but demonstrated that standardisation for age largely removed the difference. The main exception was immigrants from Scotland and Ireland, who still showed excess admission rates, largely attributable to personality disorders and alcohol problems. The diagnostic profile for immigrants from the New Commonwealth, particularly the Caribbean, was distinct, showing low rates of senile dementia and affective disorders but high rates of schizophrenia. Some of the conclusions of this widely cited study were called into question by a reanalysis of the way in which the author had handled missing data (Glover, 1987).

Dean et al (1981) explored the same issue in an opportunistic study, taking advantage of the introduction of new Mental Health Enquiry forms following the 1974 NHS reorganisation. This provided an opportunity for a publicity campaign aimed at improving data quality. For Scottish and Irish immigrants, the findings were in line with those of Cochrane. However, there was an apparent overall excess of admissions of Caribbean and African immigrants. Using Mental Health Enquiry data from the North West Thames region for the decade from 1976 to 1985 , Glover (1989b) re-examined the pattern for Caribbean immigrants. Some type of cohort phenomenon appeared to be operating. The smaller excess of overall admissions of immigrants at the earlier date was confined to male patients aged under 25. By the later date, it was apparent in males up to the age of 34 and females up to age 24 . The pattern was not confined to a single diagnostic group; it was seen most clearly in Jamaicans and not at all in Trinidadians (Glover, 1989a).

With his collaborator Bal, Cochrane (1988) revisited the issue of the relationship between mental illness and the experiences of immigrants, using data from the Mental Health Enquiry for 1981, another census year. This study explored whether the source would support the 'ethnic density' hypothesis (that the mental health of immigrants is poorer where they are in a smaller minority). By their reading, it did not. Where a pattern did emerge, the more numerous ethnic groups had higher admission rates. However, the scale at which the calculations were undertaken (NHS Regions) was substantially larger than that envisaged by the hypothesis. Cochrane reported that the data did not permit a finer-grained analysis, but the reason for this is unclear.

\section{Child development and mental illness}

Three groups have used the data from the Mental Health Enquiry to explore the relationship between aspects of birth and childhood and later development of mental illness.

The first two used Mental Health Enquiry data in conjunction with data from two of the national child development studies. These have documented prospectively the birth and progress of cohorts of children born in England in single weeks in 1946 and 1958. Members are easily identified in routine data-sets, such as the Mental Health Enquiry, by their dates of birth.

Jones et al (1994) used Mental Health Enquiry data to identify all psychiatric admissions experienced between 1974 and 1986 by members of the 1946 cohort and supplemented the study data with information from hospital case notes. Their interest was in the developmental antecedents of schizophrenia. The study demonstrated a range of developmental delays and social deficits at almost all stages in children destined to develop schizophrenia. However, these took the form of a shifted distribution of scores rather than a cluster of low scorers. This was significant in that it cast doubt on the then current hypothesis that there might be a distinct subtype of schizophrenia, characterised by severe early developmental abnormalities, possibly with a distinct aetiology.

The Medical Research Council group at Northwick Park undertook a similar study of the 1958 birth cohort (Done et al, 1991). Because of problems in data arising from the switch from Mental Health Enquiry to Hospital Episode Statistics data, this gave hospital admission data for the cohort up to age 28. From 16980 individuals, 252 were hospitalised at some time: 79 had schizophrenia, 44 had affective 
disorders and 93 had neuroses. The number with schizophrenia was close to expectation, based on contemporary estimates of the lifetime prevalence and the proportion of morbidity likely to be apparent by this age.

Using this data-set, the group explored a number of issues. They found no association between birth trauma and/or high-risk pregnancy and the development of schizophrenia. This topic had hitherto been studied mainly using the potentially biased memories of patients' parents or from inevitably incomplete collections of childbirth records that were between 20 and 40 years old. There were differences in early social adjustment between individuals who had experienced psychiatric admissions and other cohort members. By age 7 , children who subsequently developed schizophrenia showed more maladjustment than others. By 11 years of age, children who went on to develop neuroses also had increased maladjustment scores. Children who later developed affective disorders were not distinguished in this manner at either age.

O'Callaghan et al (1991) used the Mental Health Enquiry to contribute to a debate about the possible causal significance of prenatal maternal influenza in the development of schizophrenia. A sharp peak in the number of births of children who subsequently developed schizophrenia was apparent 5 months after the peak infection period of the 19571958 'Asian flu' epidemic, with an $88 \%$ increase in the numbers of first-admitted individuals born that month by comparison with the same month in the 2 previous and 2 subsequent years. By an extraordinary chance, the peak birth month identified for babies who subsequently developed schizophrenia was March 1958 - the month for which the cohort studied by the MRC group was born. In one of the most stylish ripostes in psychiatric epidemiology, Crow \& Done (1992) reported that among the 945 mothers in this cohort reporting influenza in the mid-trimester of pregnancy, there was no increase in the rate of births of children who later develop schizophrenia.

O'Callaghan et al (1994) went on to study the relationship between monthly death rates from 16 types of infectious disease and clusters of births from the group that developed schizophrenia. The only finding to appear consistently was that of an increase in such births 5 months after peaks in the death rate from broncho-pneumonia - a phenomenon they took to be associated with influenza.

\section{Health services research}

Perhaps the most obvious use for detailed statistical data about NHS mental health care is in the examination of the operation of the service itself.

\section{Bed requirements}

The earliest work on bed requirements, by Tooth \& Brooke (1961), has already been mentioned. A similar study was undertaken by Eason \& Grimes (1976) as part of the development of the 1975 White Paper Better Services for the Mentally Ill (Department of Health and Social Security, 1975). These authors were able to report on the outcome of the old longstay cohorts described in the earlier work and hence settle the controversy which had arisen subsequently about the likely course of decline of this group. In other respects, the work was similar to the earlier study.

\section{Service activity}

Madhevan \& Forster (1982) used regional Mental Health Enquiry data to compare the operation of two in-patient units based in district general hospitals in the north-east of England with that of an old mental hospital, which served both as backup to the areas served by the district general hospital units and as the sole in-patient facility for a third catchment area. They tested the somewhat simplistic null hypothesis that the distribution of age, legal status and diagnosis would be the same in each of the three units. Unsurprisingly, it was not, but rather reflected known local operational policies such as the restriction of admissions to the district general hospital units largely to working-age adults.

\section{Elderly people}

Shulman \& Arie (1978) explored the then current perception that, despite rapidly rising numbers of people aged 75 and over in the population, numbers admitted to psychiatric hospitals with a diagnosis of dementia had shown no commensurate increase. Published Mental Health Enquiry data showed that admission rates for patients aged 65 and above, which had risen in the late 1960s, had fallen in the early 1970s. The extent of the fall varied between health service regions, but it was seen in all of them. After reviewing and dismissing other possibilities, the authors concluded that at least a substantial part of the changed trend arose from a disinclination of psychiatrists to admit patients to their wards in the wake of the publication of reports on the abuse of elderly people in Ely and the Whittingham hospitals (Department of Health, 1969, 1972).

Wider patterns in the care of elderly people were studied by Hanlon et al (1998). In the early 1970s, they identified a cohort comprising about $80 \%$ of the people, aged 45-64, living in two industrial towns in west-central Scotland. They undertook initial interviews with cohort members and followed 
their subsequent health service use through routine data sources. During follow-up over 23 years, $5 \%$ of cohort members experienced admission to a psychiatric hospital. These made up one-quarter of all the hospital bed-days used by the cohort. Affective psychoses and other depressive disorders accounted for about $35 \%$ of the admissions, but senile and presenile organic conditions comprised $11.5 \%$ of the admissions and accounted for $24 \%$ of the bed-days.

\section{Performance indicators}

The Department of Health uses hospital activity data in the production of many national performance indicators. Because the data underlying these indicators are available in detailed format, it is possible to explore the appropriateness of the chosen formulation. Glover has reported two studies of this type. The first (Glover et al, 1990) examined indicators of stay length and the decision to use grouped lengths of stay rather than to consider this as a continuously varying parameter. The second (Glover \& Bindman, 2001) explored a proposal to establish an indicator of out-of-area admissions using Hospital Episode Statistics data. In both cases, it was possible to show that the immediately intuitive formulation chosen by the Department of Health had unforeseen pitfalls.

\section{Geographic variations in service use}

A preoccupation of health service researchers in the 1990s was the pattern of geographical variation in rates of mental illness, and resulting service needs. This has implications for distribution of financial resources as well as for research and clinical audit. Mathematical modelling of these patterns requires the type of detailed national data available from the Hospital Episode Statistics or the Mental Health Enquiry.

Four teams have published in this area. Jarman et al (1992) undertook the least sophisticated exploration. Thornicroft (1991) invoked a range of concepts from social geography to develop models accounting for the observed variation but did not go on to develop a useable index of needs. Smith et al (1996) developed a new mental health resource allocation formula for the Department of Health. Their modelling strategy attempted to encompass the impact of varying levels of service provision on the proportion of psychiatric morbidity, which would translate into admission activity, although doubts were raised about the extent to which aspects of their data analysis reflected real patterns of clinical organisation (Lelliott et al, 1996). Finally, Glover et al (1998) developed an index designed to be easy to calculate for any area of the country and produced supporting computer software.

\section{Discussion}

The comprehensive coverage, large case numbers and raw-data format of the Mental Health Enquiry and Hospital Episode Statistics have made them valuable to researchers, planners and policy-makers. This is demonstrated by the wide range of uses to which they have been applied (Box 2) and also the perspectives of workers using them.

It is inevitable that there are many problems associated with the use of these data sources. The scale and complexity of the task of gathering and collating the data, the large numbers of individuals involved and the periodic reorganisations inevitably give rise to poorer levels of completeness and accuracy than would be expected in data collected for research projects. However, these are inevitable concomitants of the breadth and time-span which give them their value. It has been argued that refined levels of accuracy may not matter in large data-sets if questions are formulated with intelligent recognition of the strengths and weaknesses of the source (Yates, 1982).

The raw-data format used (data are stored as individual records for each admission) gives these data absolute flexibility in analysis. In analyses of geographical distribution, data can be selected that follow the constantly changing pattern of England's administrative geography. Records can be linked to data about the same individuals from other sources, as in the birth cohort studies. The advent of the new NHS number should make this technically easier, although the constraints of data protection legislation are becoming increasingly exacting. As a comprehensive system for collecting data on a national scale, hospital admission data are the

\section{Box 2 Research uses of in-patient statistics}

Investigations of patterns of mental illness in relation to:

- deprivation

- ethnicity

- social and occupational groups

- time (secular trends)

Studying the relation of mental illness to:

- developmental characteristics

- aspects of pregnancy and childbirth

The impact of new treatment methods

Changes in the use of mental health services

Bed requirements 
ultimate test of whether new drugs or treatment approaches have had the intended impact at the level of the whole population. The public availability of the data also means that researchers can, and do, reanalyse the work of others, permitting debates to be taken further than is usually possible.

However, with the diminishing role of hospital admission in the overall pattern of mental health care, the relevance of statistics limited to admissions to hospital, in isolation from other types of mental health care, has diminished. Admission statistics are increasingly inadequate as proxies for patterns of psychotic illness in the population. Recent large psychiatric morbidity surveys (Meltzer et al, 1995) do not make up this deficit, first because of their infrequency and second because the number of cases of these severe forms of mental illness, even in samples of 10000 or so, is too small to provide detail of patterns across geographical areas or age, gender and ethnic groups. For this type of work, comprehensive statistics are always likely to be required.

The new Mental Health Minimum Data Set will document periods of care by specialist mental health services, in whatever context these are provided (Glover, 2000). This will not remove the usefulness of hospitalisation statistics, as this type of care will continue to be of particular interest, being clinically important, expensive and invested with great symbolic significance in the public perception of the position in society of those with mental illnesses. Although often derided for their imperfections, this article has shown that such data have produced many important insights into patterns of mental illness and mental health care which could not have been reached any other way. For all their faults, they are simply irreplaceable.

\section{References}

Brooke, E. M. (1963) A Cohort Study of Patients First Admitted to Mental Hospitals in 1954 and 1955. London: Stationery Office.

Cochrane, R. (1977) Mental illness in immigrants to England and Wales: an analysis of mental hospital admissions, 1971. Social Psychiatry, 12, 25-35.

— \& Bal, S. S. (1988) Ethnic density is unrelated to incidence of schizophrenia. British Journal of Psychiatry, 153, 363366.

Cooper, J. E. (1967) Diagnostic change in a longitudinal study of psychiatric patients. British Journal of Psychiatry, 113, 129-142.

Crow, T. J. \& Done, D. J. (1992) Prenatal exposure to influenza does not cause schizophrenia. British Journal of Psychiatry, 161, 390-393.

Dean, G., Walsh, D., Downing, H., et al (1981) First admissions of native-born and immigrants to psychiatric hospitals in south-east England 1976. British Journal of Psychiatry, 139, 506-512.

Department of Health (1962) A Hospital Plan for England and Wales. Cmnd 1604. London: HMSO.

- (1969) Report of the Committee of Inquiry into Allegations of Ill-treatment, opf patients and other irregularities at the Ely Hospital, Cardiff. Cmnd 3975. London: HMSO.
- (1972) Report of the Committee of Inquiry into Whittingham Hospital. Cmnd 4861. London: HMSO.

Department of Health and Social Security (1975) Better Services for the Mentally Ill. London: Department of Health and Social Security.

Der, G., Gupta, S. \& Murray, R. M. (1990) Is schizophrenia disappearing? Lancet, 335, 513-516.

Done, D. J., Johnstone, E. C., Frith, C. D., et al (1991) Complications of pregnancy and delivery in relation to psychosis in adult life: data from the British perinatal mortality survey sample. BMJ, 302, 1576-1580.

Eason, R. J. \& Grimes, J. A. (1976) In-patient care of the mentally ill: a statistical study of future provision. Health Trends, 8, 13-18.

Forster, D. P. \& Madhevan, S. (1981) Information sources for planning and evaluating adult psychiatric services. Community Medicine, 3, 160-168.

Gandy, R. J. (1979) An enquiry into the Mental Health Enquiry. British Journal of Psychiatry, 135, 531-534.

Glover, G. R. (1987) 993W: birthplace not stated or born at sea. Psychological Medicine, 17, 1009-1012.

- (1989a) Differences in psychiatric admission patterns between Caribbeans from different islands. Social Psychiatry and Psychiatric Epidemiology, 24, 209-211.

- (1989b) The pattern of psychiatric admissions of Caribbean-born immigrants in London. Social Psychiatry and Psychiatric Epidemiology, 24, 49-56.

- (2000) A comprehensive clinical database for mental health care in England. Social Psychiatry and Psychiatric Epidemiology, 35, 523-529.

- \& Bindman, J. (2001) Out of area hospitalisations - the view from current routine statistics. Psychiatric Bulletin, $25,376-378$

-, Preston, D. \& Farmer, R. (1990) Indicators of mental hospital bed use. Health Trends, 22, 111-115.

-, Robin, E. Emami, J., et al (1998) A needs index for mental health care. Social Psychiatry and Psychiatric Epidemiology, 33, 89-96.

Goldacre, M., Shiwach, R. \& Yeates, D. (1994) Estimating incidence and prevalence of treated psychiatric disorders from routine statistics: the example of schizophrenia in Oxfordshire. Journal of Epidemiology and Community Health, 48, 318-322.

Hanlon, P., Walsh, D., Whyte, B., et al (1998) Hospital use by an ageing cohort: an investigation into the association between biological, behavioural and social risk markers and subsequent hospital utilisation. Journal of Public Health Medicine, 20, 467-476.

Jarman, B., Hirsch, S., White, P., et al (1992) Predicting psychiatric admission rates. BMJ, 304, 1146-1151.

Jones, P., Rodgers, B., Murray, R., et al (1994) Child development risk factors for adult schizophrenia in the British 1946 birth cohort. Lancet, 344, 1398-1402.

Korner, E. (1982) National Health Service and Department of Health and Social Security Steering Group on Health Services Information, First Report. London: National Health Service \& Department of Health and Social Services.

Lelliott, P., Jarman, B., Bajekal, M., et al (1996) Commentaries on: An index of need for psychiatric services based on inpatient utilisation. British Journal of Psychiatry, 169, 317321.

Madhevan, S. \& Forster, D. P. (1982) Psychiatric units in district general hospitals and traditional mental hospitals: some recent evidence. British Journal of Psychiatry, 140, $160-165$.

Meltzer, H., Gill, B., Petticrew, M., et al (1995) OPCS Surveys of Psychiatric Morbidity in Great Britain, Report 1: The Prevalence of Psychiatric Morbidity Among Adults Living in Private Households. London: Stationery Office.

Ministry of Health (1969) Psychiatric Hospitals and Units in England and Wales. In-patient Statistics from the Mental Health Enquiry for the Years 1964, 1965 and 1966. London: Stationery Office.

O'Callaghan, E., Sham, P., Takei, N., et al (1991) Schizophrenia after prenatal exposure to 1957 A2 influenza epidemic. Lancet, 337, 1248-1250. 
-, Sham, P. C., Takei, N., et al (1994) The relationship of schizophrenic births to 16 infectious diseases. British Journal of Psychiatry, 165, 353-356.

Price, J. S., Slater, E. \&. Hare, E. H (1971) Marital status of first admissions to psychiatric beds in England and Wales in 1965 and 1966. Social Biology, 18, S74-S94.

Registrar General (1953) Registrar General's Statistical Review of England and Wales for the Year 1949: Supplement on General Morbidity, Cancer and Mental Health. London: Stationery Office.

- (1955) Registrar General's Statistical Review of England and Wales for the Two Years 1950-1951; Supplement on General Morbidity, Cancer and Mental Health. London: Stationery Office.

- (1958) Registrar General's Statistical Review of England and Wales for the Two Years 1952-1953; Supplement on Mental Health. London: Stationery Office.

- (1960) Registrar General's Statistical Review of England and Wales for the Three Years 1954-1956; Supplement on Mental Health. London: Stationery Office.

- (1961) Registrar General's Statistical Review of England and Wales for the Two Years 1957-1958; Supplement on Mental Health. London: Stationery Office.

- (1962) Registrar General's Statistical Review of England and Wales for the Year 1959: Supplement on Mental Health. London: Stationery Office.

Shulman, K. \& Arie, T. (1978) Fall in admission rate of old people to psychiatric units. BMJ, 1, 156-158.

Smith, P., Sheldon, T. A. \& Martin, S. (1996) An index of need for psychiatric services based on in-patient utilisation. British Journal of Psychiatry, 169, 308-316.

Symonds, R. L. \& Williams, P. (1981) Lithium and the changing incidence of mania. Psychological Medicine, 11, 193-196.

Thornicroft, G. (1991) Social deprivation and rates of treated mental disorder. Developing statistical models to predict psychiatric service utilisation. British Journal of Psychiatry, 158, 475-484.

Tooth, G. C. \& Brooke, E. M. (1961) Trends in the mental hospital population and their effect on future planning. Lancet, i, 710-713.

Yates, J. (1982) Hospital Beds: A Problem for Diagnosis and Management. London: Heinemann Medical Books.

\section{Multiple choice questions}

1 Studies have shown that:

a more than $40 \%$ of diagnoses in routine statistics are wrong

b more than $80 \%$ of patients with schizophrenia will receive in-patient care within 5 years

c Scottish and Irish people living in England have low mental hospital admission rates

d admission profiles for immigrants from the Caribbean in England vary between groups from different islands

e in the 1950s, alcohol problems in men were most common in social class I.

2 Mental hospital admission statistics:

a are currently collected by the Office of National Statistics

b can be used to show variations in admission rate in different parts of the country

c were linked to form a patient-based admission register in the mid-1950s

d suggest a decline in the incidence of schizophrenia in England in the late 1970s e showed a rapid decline in admissions for manicdepressive illness following the introduction of lithium treatment.

3 Records from the Mental Health Enquiry:

a were first sent to the Department of Health and Social Security in computerised form in 1974

b were first collected as a result of the 1930 Mental Treatment Act

c have been used to study the relationship between complications of pregnancy and childbirth and the development of schizophrenia

d appear to show a relationship between the 1957-1958 Asian flu epidemic and the increased number of births of children who subsequently developed schizophrenia

e originally included a question about whether the patient was related by blood to their first spouse.

\section{Mental Health Enquiry data showed that:}

a without a firm government commitment to deinstitutionalisation, mental hospital populations were likely to go on rising

b admission rates of people with dementia seemed to fall in the wake of reports of abuse and neglect of hospital patients

c within social classes, higher levels of education were associated with more mental illness

d the invention of chlorpromazine in the early 1950s was associated with a reduction in mental hospital readmission rates

e of a cohort of all the people born in England in a single week in 1958 , by age 28 , more had been admitted with schizophrenia than with an affective disorder.

5 In routine data sources:

a data quality is a constant concern

b raw-data sources can be more flexibly analysed than tabulated data

c inaccuracies in diagnoses come both from the coding process and clinician errors

d population surveys will soon become the preferred method for estimating the prevalence of schizophrenia

e patient-based linkage is impossible without computers. 